\title{
Engineering Escherichia coli to convert acetic acid to $\beta$-caryophyllene
}

\author{
Jianming Yang ${ }^{1,2^{*}}$ and Qingjuan $\mathrm{Nie}^{3}$
}

\begin{abstract}
Background: Under aerobic conditions, acetic acid is the major byproduct produced by E. coli during the fermentation. And acetic acid is detrimental to cell growth as it destroys transmembrane pH gradients. Hence, how to reduce the production of acetic acid and how to utilize it as a feedstock are of intriguing interest. In this study, we provided an evidence to produce $\beta$-caryophyllene by the engineered $E$. coli using acetic acid as the only carbon source.

Results: Firstly, to construct the robust acetate-utilizing strain, acetyl-CoA synthases from three different sources were introduced and screened in the E. coli. Secondly, to establish the engineered strains converting acetic acid to $\beta$-caryophyllene, acetyl-CoA synthase (ACS), $\beta$-caryophyllene synthase (QHS1) and geranyl diphosphate synthase (GPPS2) were co-expressed in the E. coli cells. Thirdly, to further enhance $\beta$-caryophyllene production from acetic acid, the heterologous MVA pathway was introduced into the cells. What's more, acetoacetyl-CoA synthase (AACS) was also expressed in the cells to increase the precursor acetoacetyl-CoA and accordingly resulted in the increase of $\beta$-caryophyllene. The final genetically modified strain, YJM67, could accumulate the production of biomass and $\beta$-caryophyllene up to 12.6 and $1.05 \mathrm{~g} / \mathrm{L}$ during $72 \mathrm{~h}$, respectively, with a specific productivity of $1.15 \mathrm{mg} \mathrm{h}^{-1} \mathrm{~g}^{-1}$ dry cells, and the conversion efficiency of acetic acid to $\beta$-caryophyllene (gram to gram) reached $2.1 \%$. The yield of $\beta$-caryophyllene on acetic acid of this strain also reached approximately $5.6 \%$ of the theoretical yield.

Conclusions: In the present study, a novel biosynthetic pathway for $\beta$-caryophyllene has been investigated by means of conversion of acetic acid to $\beta$-caryophyllene using an engineered Escherichia coli. This was the first successful attempt in $\beta$-caryophyllene production by E. coli using acetic acid as the only carbon source. Therefore, we have provided a new metabolic engineering tool for $\beta$-caryophyllene synthesis.
\end{abstract}

Keywords: $\beta$-caryophyllene, Acetic acid, MVA pathway, E. coli

\section{Background}

$\beta$-caryophyllene, a common sesquiterpene that is widely found in plants $[1,2]$, is being considered for use as a component in the next generation aircraft fuel [3-5]. Conventionally, like other sesquiterpenes, extraction from plants and chemical synthesis have always been accepted as the common options for the production of $\beta$-caryophyllene on a large scale, yet, both methods have their own disadvantages. Low concentration and poor recovery yields $[6,7]$ make the isolation of $\beta$-caryophyllene from plants

\footnotetext{
*Correspondence: yjming888@126.com

2 Key Lab of Applied Mycology, College of Life Sciences, Qingdao

Agricultural University, No.700 Changcheng Road, Chengyang District, Qingdao 266109, China

Full list of author information is available at the end of the article
}

both infeasible and uneconomical. Meanwhile, complexity of the process, the diminishing reserve of petroleum and increasingly serious environmental problems are impelling us to move our eyes on the microbial production of $\beta$-caryophyllene, which can utilize renewable glucose derived from lignocellulose. In addition, compared with traditional methods, $\beta$-caryophyllene produced by microbial fermentation expects to be a good alternative mainly due to its environmental friendliness and sustainable development.

However, in spite of the above advantages in the microbial synthesis, there are also some bottlenecks limiting its application. For instance, under aerobic conditions, acetic acid (HAc) is the major byproduct produced during fermentation in high cell density cultures. Unfortunately, high concentration of HAc is detrimental to cell 
growth as it destroys transmembrane $\mathrm{pH}$ gradients. This negatively affects internal osmotic pressure, recombinant protein synthesis and biomass formation $[8,9]$. In addition, the production of the byproduct HAc accordingly decreases the carbon effective utilization and the yield of target product.

In the previous studies, acetic acid had been considered to be used as a feedstock for production of biofuel or biochemical since it could be generated from a variety of cheap sources. (1) Methanol carbonylation is an effective method for HAc production [10]. (2) HAc is a major product during syngas fermentation $[11,12]$ and a byproduct from hydrolysis (under acid or alkali pretreatment [13] or pyrolysis of lignocellulosic biomass [14]. (3) Methane from natural gas or biogas can be converted to HAc [15]. (4) HAc is also an intermediate from anaerobic digestion of organic wastes. [16].Cryptococcus curvatus had been demonstrated to use HAc as a major carbon source for lipid production [17-19] and some Clostridium species could utilize HAc and sugar concurrently for alcohols or butyrate biosynthesis [20, 21]. Hence, strengthening the ability of $E$. coli to assimilate HAc would lessen harmful effects of HAc, recycle wasted carbon, and enhance carbon flux toward the desired pathways. However, there are no reports on metabolic engineering of the $E$. coli strain which can utilize HAc as the main carbon source to produce $\beta$-caryophyllene.

In the present study, we are making an attempt to use acetic acid (HAc) as a feedstock for production of $\beta$-caryophyllene. A multi-step metabolic engineering strategy (Fig. 1) was employed to enhance the ability to utilizing HAc, increase the supply of some precursors such as IPP, DMAPP, GPP and acetoacetyl-CoA, which ultimately led to the increase in the $\beta$-caryophyllene production. The final genetically modified strain, YJM67, cultured under the fed-batch fermentation condition, could accumulate the yield of biomass and $\beta$-caryophyllene up to 12.6 and $1.05 \mathrm{~g} / \mathrm{L}$ during $72 \mathrm{~h}$, respectively, and the conversion efficiency of HAc to $\beta$-caryophyllene (gram to gram) reached $2.1 \%$, which was the first successful attempt in $\beta$-caryophyllene production by $E$. coli using the HAc as the only carbon source. Therefore, we have provided a new metabolic engineering tool for $\beta$-caryophyllene synthesis.

\section{Results and discussion \\ Engineered the high efficiency pathway for HAc utilization in E. coli}

HAc is employed as a potential substrate for production of biomass, biofuels and value added chemicals. However, E. coli has a restricted ability to use HAc for biomass growth. The metabolic pathways which $E$. coli utilizes HAc are mainly via AMP-forming acetyl-CoA synthetase (the acs pathway) and phosphotransacetylase/ acetate kinase (the reversible PTA-ACKA pathway) [9, $22]$. In the previous study, it has proven that overexpressing the single acs gene along with maintaining the native HAc pathways was the best strategy for HAc assimilation in E. coli $[23,24]$. A suitable method to optimize pathway efficiency may be to use genes from different organisms [25]. In this paper, the acetyl-CoA synthase enzymes from native E. coli, Salmonella typhimurium LT2 and Acetobacter pasteurianus were assessed to utilize HAc for cell growth.

Therefore, in this paper, to construct the acetate utilization pathway in $E$. coli, the different $A C S$ genes were screened. The gene $A C S_{E C}$ from E. coli, $A C S_{S T}$ from $S$. typhimurium LT2 and $A C S_{A P}$ from $A$. pasteurianus

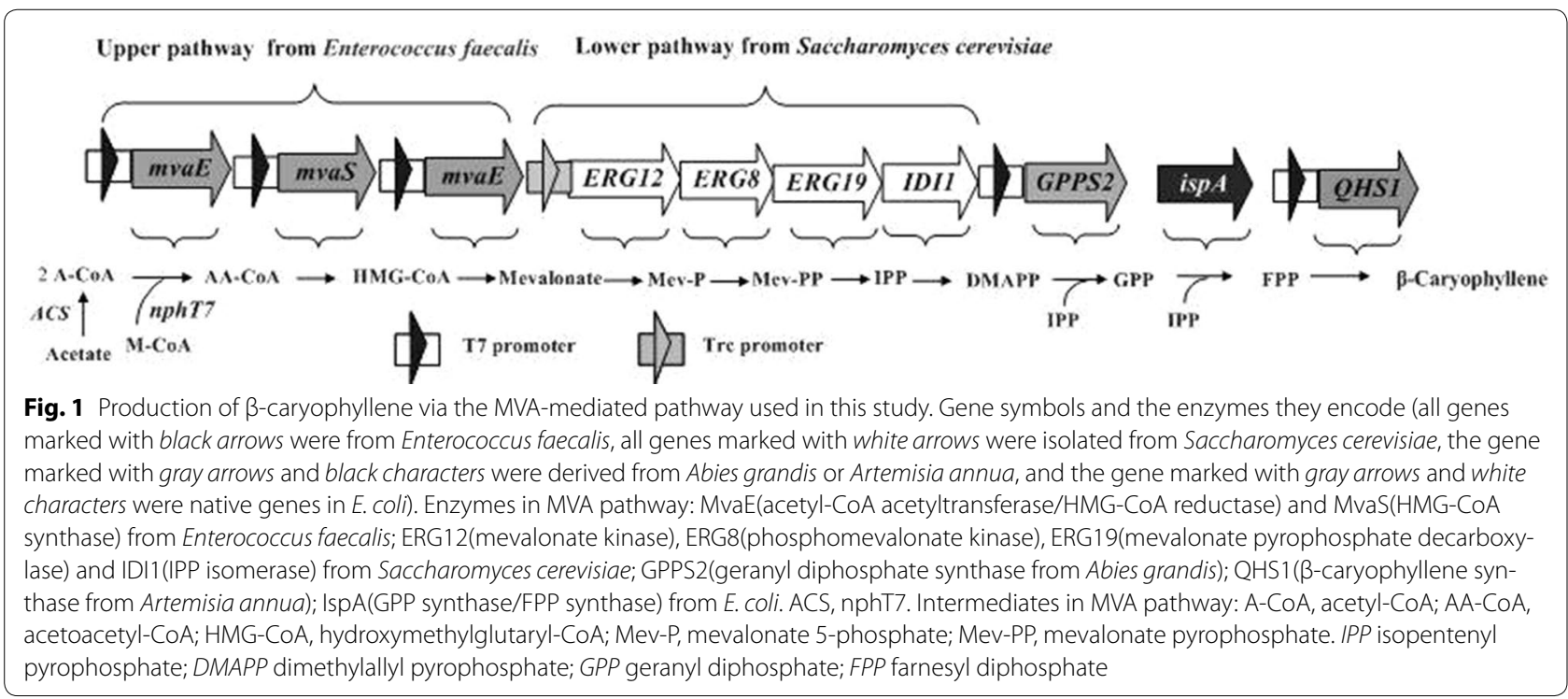


were cloned into the plasmid pCOLDuet- 1 forming the plasmids pYJM60, pYJM61 and pYJM62, respectively. The different strains containing pYJM60, pYJM61 and pYJM62 named as YJM60, YJM61 and YJM62, were cultured using M9 medium with different concentrations of sodium acetate. As shown in Fig. 2, the results showed that the strain containing $A C S_{A P}$ gene grow best among three strains, also suggested that the enzyme from $A$. pasteurianus was the most efficient in the conversion of acetate into acetyl-CoA.

\section{Biosynthesis of $\beta$-caryophyllene from HAc using native MEP pathway}

Farnesyl diphosphate (FPP), generated from either the DXP or MVA pathway [26, 27], could be catalyzed by the $\beta$-caryophyllene synthase into $\beta$-caryophyllene $[28$,
29]. Nevertheless, E. coli harbors MEP pathway, and is not capable of producing $\beta$-caryophyllene on its own due to the lack of $\beta$-caryophyllene synthase. In the study, to produce $\beta$-caryophyllene, the optimized QHS1 gene from Artemisia annua was overexpressed along with $\mathrm{ACS}_{\mathrm{AP}}$ enzyme from $A$. pasteurianus in the engineered E. coli (YJM63 containing pACY-QHS1/pCOL-ACS ${ }_{A P}$ ). Firstly, the recombinant strain YJM63 was grown in the M9 medium with acetate as the carbon source, and the target product $\beta$-caryophyllene was not detected. While the engineered strain cultured in the M9 medium with $2 \mathrm{~g} / \mathrm{L}$ glucose as the carbon source, it could produce $56 \pm 6 \mu \mathrm{g} / \mathrm{L} \beta$-caryophyllene. No $\beta$-caryophyllene production was detected in the control strain harboring the

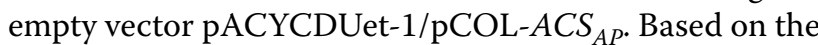
results, using the native MEP pathway, QHS1 gene from
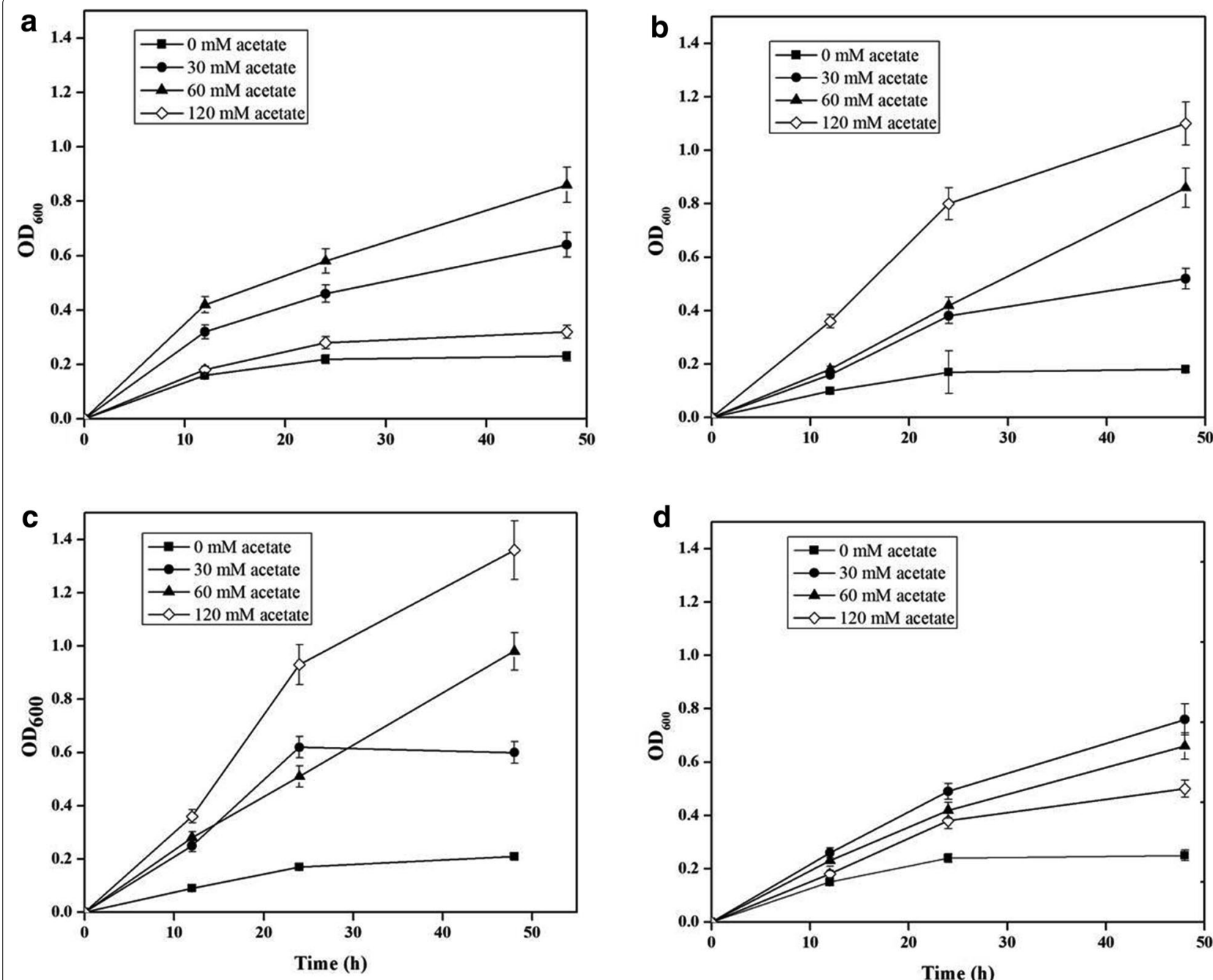

Fig. 2 Comparative growths of the engineered strains using acetic acid. The engineered strains E. coli BL21(DE3)/pCOLADUet-1 (a), BL21(DE3)/ pYJM60 (b), BL21(DE3)/pYJM62 (c) and BL21(DE3)/pYJM61 (d) grew on different concentrations of acetate. The OD 600 was measured spectrophotometrically. The experiment was performed in triplicate 
A. annua and $A C S_{A P}$ gene from A. pasteurianus, a novel biosynthetic pathway for the $\beta$-caryophyllene production with acetate as the carbon source has been successfully established in the E. coli. However, the effectiveness of the whole metabolic pathway for $\beta$-caryophyllene production is too low. The main reason might be short of other important precursors such as DMAPP, IPP and GPP.

Geranyl diphosphate (GPP), the key precursor of sesquiterpene production, is catalyzed from the condensation of dimethylallyl diphosphate and isopentenyl diphosphate by GPP synthase [30]. In this paper, to enhance the supply of GPP and accordingly to increase $\beta$-caryophyllene production, the GPP synthase of Abies grandis was co-expressed in the cell along with QHS1 and $A C S_{A P}$ genes and formed into the engineered strain YJM64 (E. coli BL21(DE3) containing pACY-QHS1-GPPS2/pCOL-ACS $A P$ ). According to the GC analysis results, after $24 \mathrm{~h}$ of culture, $102 \pm 9 \mu \mathrm{g} / \mathrm{L}$ $\beta$-caryophyllene was produced by the $E$. coli strain YJM64. The results demonstrated that the heterologous expression of GPP synthase (GPPS) is beneficial to the $\beta$-caryophyllene production. This finding is corresponding to previous studies on the production of other terpenes such as $\alpha$-pinene and sabinene [31, 32].

\section{Establishing a MVA-mediated biosynthetic pathway for $\beta$-caryophyllene production from acetate}

Despite of progress made in the $\beta$-caryophyllene biosynthesis using the native MEP pathway, the yield of $\beta$-caryophyllene was so low that it is not economic and feasible for industrial application. In previous studies, the hybrid MVA pathway has proven to be efficient on the biosynthesis of DMAPP and IPP [33, 34] which are the precursors of all terpenes. Based on these findings, a hypothesis can be put forward that the MVA pathway might be more efficient than the MEP pathway in $\beta$-caryophyllene production using acetate as carbon source.

In an attempt to increase the production of $\beta$-caryophyllene, the hybrid MVA pathway was introduced into $E$. coli and overexpressed along with QHS1 from $A$. annua, GPPS2 gene from A. grandis and $A C S_{A P}$ gene from $A$. pasteurianus. As expected, the recombinant strain YJM66 (pACY-mvaE-mvaS-QHS1-GPPS2/ pTrc-Low/pCOL- $A C S_{A P}$ ) carrying hybrid MVA pathway could accumulate $8 \pm 0.75 \mathrm{mg} / \mathrm{L} \beta$-caryophyllene, which is about eight-fold higher than that $(102 \pm 9 \mu \mathrm{g} / \mathrm{L})$ produced by the control strain YJM64 (pACY-QHS1GPPS $2 / \mathrm{pCOL}-A C S_{A P}$ ) without the hybrid MVA pathway. According to the data obtained, it may be easier to make a conclusion that the hybrid MVA pathway is conducive to the $\beta$-caryophyllene biosynthesis.

\section{The effect of AACS on $\beta$-caryophyllene production from Acetate}

In recent studies, acetoacetyl-CoA synthase (AACS) from Streptomyces sp. strain CL190 has been proven to catalyze a single condensation of acetyl-CoA and malonyl-CoA to form acetoacetyl-CoA [35]. Unlike acetoacetyl-CoA thiolase (EC 2.3.1.9), which also synthesize acetoacetyl-CoA by reversible nondecarboxylative condensation of two molecules of acetyl-CoA and prefers acetoacetyl-CoA thiolysis to acetoacetyl-CoA synthesis, NphT7 proves no thiolysis activity against acetoacetylCoA, and since NphT7-catalyzed acetoacetyl-CoA synthesis is essentially an energy-favored reaction, NphT7 could be an ideal enzyme to supply acetoacetyl-CoA in cells [36].

When the AACS-encoding gene (nphT7) was expressed combining with the HMG-CoA synthase gene and the HMG-CoA reductase gene in E.coli, the engineered strain could achieve 3.5 -fold higher production of mevalonate than the control strain without nphT7 expression [36]. Based on these findings, it may be hypothesized that overexpression of acetoacetyl-CoA synthase (AACS) in $\beta$-caryophyllene-producing strain would enhance $\beta$-caryophyllene productivity.

In the paper, to further increase $\beta$-caryophyllene production, the AACS-encoding gene (nphT7) was overexpressed in the $\beta$-caryophyllene producing E. coli YJM67 (pACY-mvaE-mvaS-QHS1-GPPS2/pTrc-Low/pCOL$A C S_{A P}$-nphT7). After cultured in the M9 medium with $60 \mathrm{mM}$ acetate as the carbon source for $24 \mathrm{~h}$, the yield of $\beta$-caryophyllene reached $22 \pm 1.8 \mathrm{mg} / \mathrm{L}$, which is 2.75 fold to the control strain YJM66 without nphT7 gene expression $(8 \pm 0.75 \mathrm{mg} / \mathrm{L})$. The results demonstrated that acetoacetyl-CoA synthase is helpful to increase acetoacetyl-CoA in cells and accordingly to enhance $\beta$-caryophyllene production.

\section{Effect of the type and concentration of the nitrogen source on $\beta$-caryophyllene production}

The source of the nitrogen in the medium plays an important role in improving the biosynthesis of desired product $[37,38]$. To investigate the effect of type and concentration of organic nitrogen source on $\beta$-caryophyllene production, six different organic nitrogen sources were chosen and valuated (Fig. 3a). Among the organic nitrogen supplements tried, the Solarbio beef extract allowed a significantly higher $\beta$-caryophyllene production than the other organic nitrogen sources.

Then to determine the optimum concentration of Solarbio beef extract, various beef extract concentrations, ranging from 1 to $15 \mathrm{~g} / \mathrm{L}$, were tested. According to the data shown in Fig. 3b, the maximum production of $\beta$-caryophyllene reached $106 \pm 9.6 \mathrm{mg} / \mathrm{L}$, which was 

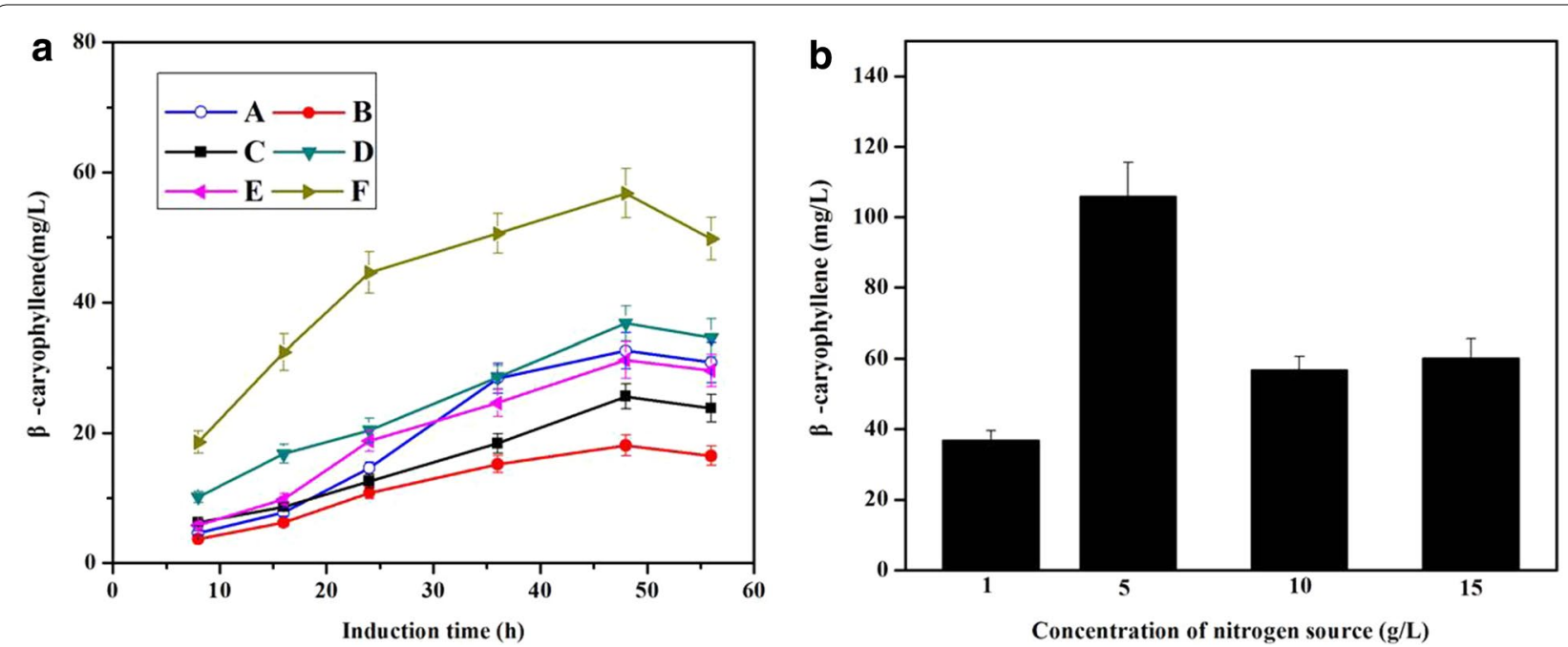

Fig. 3 The effects of types and concentrations of nitrogen source on $\beta$-caryophyllene production by YJM67. a The Effect of different organic nitrogen source on $\beta$-caryophyllene production by YJM67. a Beef extract (Aladdin, O); b yeast extract powder (Beijing AoBoXing Bio-Tech Co., Ltd, $\mathbf{Q}$ ); $\mathbf{c}$ beef extract powder (MDBio, Inc, $\mathbf{\square}$ ); d beef extract (Beijing Shuangxuan Microbe Culture Medium Products Factory, $\boldsymbol{\nabla}$ ); e beef extract (Sinopharm Chemical Reagent Co., Ltd, 4$) ; \mathbf{f}$ beef extract (solarbio, - ) a The Effect of concentration of nitrogen source on $\beta$-caryophyllene production by YJM67. When $\mathrm{OD}_{600}$ reached 0.6-0.9, cultures were induced for $56 \mathrm{~h}$ using IPTG in shake-flasks. All the experiments were carried out in triplicates. Optimized conditions: Nitrogen sources, beef power; concentrations of nitrogen source, $5 \mathrm{~g} / \mathrm{L}$

approximately five times as much as the production before optimization $(22 \pm 1.8 \mathrm{mg} / \mathrm{L})$.

Based on the above data, the most suitable types and concentration of nitrogen source for $\beta$-caryophyllene production using the engineered strain YJM67 were $5 \mathrm{~g} / \mathrm{L}$ Solarbio beef extract.

\section{Lab-scale batch production of $\beta$-caryophyllene}

To scale up $\beta$-caryophyllene production from HAc, a $\mathrm{pH}$-coupled HAc fed batch fermentation, which can gradually add HAc to the fermentation medium, was performed in a 5-L-scale laboratory batch reactor, in which was grown our most optimized strain, YJM67. Based on the flask condition, the final engineered strain YJM67 was cultured in the 5-L-scale laboratory batch reactor with the minimal medium plus $5 \mathrm{~g} / \mathrm{L}$ beaf extract. As seen in the Fig. 4, the production of biomass and $\beta$-caryophyllene increased substantially without obvious lag phase and their maximum concentrations reached 12.6 and $1.05 \mathrm{~g} / \mathrm{L}$ at $72 \mathrm{~h}$, respectively, with a specific productivity of $1.15 \mathrm{mg} \mathrm{h}^{-1} \mathrm{~g}^{-1}$ dry cells, and the conversion efficiency of HAc to $\beta$-caryophyllene (gram to gram) reached $2.1 \%$. The yield of $\beta$-caryophyllene on HAc of this strain also reached approximately $5.6 \%$ of the theoretical yield (of $37.81 \%$ ) based on the following formula: $9 \mathrm{HAc} \rightarrow 9$ Acetyl-CoA $\rightarrow \beta$-caryophyllene.

These results suggest great potential for this engineered $E$. coli strain in the production of $\beta$-caryophyllene on a large scale.

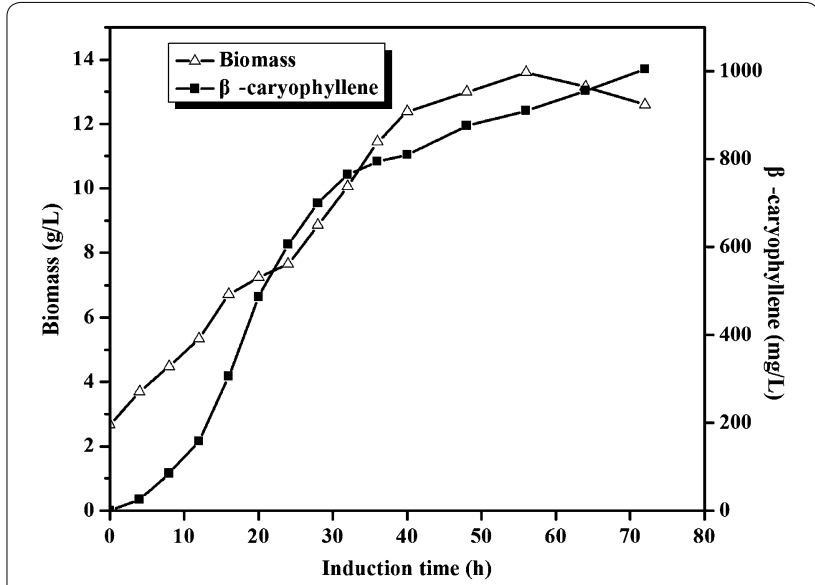

Fig. 4 The time course of $\beta$-caryophyllene production by YJM67. Biomass $(\Delta)$ and $\beta$-caryophyllene accumulation $(\boldsymbol{\square})$ in YJM67. Induction was carried out when $\mathrm{OD}_{600}$ reached about 6 at $30^{\circ} \mathrm{C}$. Other experimental conditions are described in section "Fed-Batch Fermentation"

\section{Conclusions}

In the paper, a novel biosynthetic pathway for $\beta$-caryophyllene has been constructed by assembling acetyl-CoA synthase, acetoacetyl-CoA synthase, $\beta$-caryophyllene synthase and the hybrid MVA pathway in the engineered $E$. coli strain. The final genetically modified strain, YJM67, could accumulate the production of biomass and $\beta$-caryophyllene up to 12.6 and $1.05 \mathrm{~g} / \mathrm{L}$ during $72 \mathrm{~h}$, respectively, and the conversion efficiency of 
HAc to $\beta$-caryophyllene (gram to gram) reached $2.1 \%$. To our knowledge, this was the first successful attempt in $\beta$-caryophyllene production by $E$. coli using the HAc as the only carbon source. Therefore, we have opened up a way to produce $\beta$-caryophyllene or other biochemicals and bioenergy on the HAc replacing glucose as a carbon source.

\section{Methods}

\section{Strains, plasmids and culture conditions}

All strains and plasmids used in this study are listed in Table 1. E. coli DH5a was employed in all the cloning work. E. coli BL21(DE3) was used for the product biosynthesis. For $\beta$-caryophyllene production, different strains were cultivated in shake-flask or fed-batch fermentation conditions with modified M9 minimal medium containing different concentrations of sodium acetate, beef extract $5 \mathrm{~g} / \mathrm{L}$. M9 minimal medium consists of $\mathrm{Na}_{2} \mathrm{HPO}_{4}$ 6, $\mathrm{KH}_{2} \mathrm{PO}_{4} 3, \mathrm{NH}_{4} \mathrm{Cl} 1, \mathrm{NaCl} 0.5, \mathrm{MgSO}_{4} 0.24 \mathrm{~g} / \mathrm{L}$. If necessary, suitable antibiotics were added to the culture medium at the following concentrations: ampicillin $(100 \mu \mathrm{g} / \mathrm{ml})$, kanamycin $(50 \mu \mathrm{g} / \mathrm{ml})$, and chloramphenicol $(34 \mu \mathrm{g} / \mathrm{ml})$.

\section{Plasmid construction}

The nucleotide sequence of acetyl-CoA synthetase genes from E. coli $\left(A C S_{E C}\right.$, GenBank No. ACB05066.1) was obtained by PCR amplification using its genomic DNA as the template. The nucleotide sequences of acetyl-CoA synthetase genes from Salmonella typhimurium LT2 $\left(A C S_{S T}\right.$, GenBank No. AAL23099.1) and from Acetobacter pasteurianus $\left(A C S_{A P}\right.$, GenBank No. AKR48484.1) were chemically synthesized by Genray Company with plasmid pGH as the vector (called pGH-ACS $S_{S T}$ and called pGH- $A C S_{A P}$ ).

The nucleotide sequence of acetoacetyl CoA synthase from Streptomyces sp. CL190 (nphT7, GenBank No. AB540131.1) was chemically synthesized by Genray Company with plasmid pGH as the vector (called pGH- nphT7).

The nucleotide sequences of $\beta$-caryophyllene synthase genes from Artemisia annua (QHS1, GenBank No. AF472361.1) was analyzed using online software (http:// www.genscript.com/cgi-bin/tools/rare_codon_analysis) and optimized to the preferred codon usage of E. coli (http://www.jcat.de/). The codon-optimized QHS1 gene was chemically synthesized by Genray Company with plasmid pGH as the vector (called pGH-QHS1).

Table 1 Strains and plasmids used in this study

\begin{tabular}{|c|c|c|}
\hline Name & Relevant characteristics & References \\
\hline \multicolumn{3}{|l|}{ Strains } \\
\hline E.coli BL21(DE3) & $\mathrm{F}^{-}$ompThsdS $_{\mathrm{B}}\left(\mathrm{r}_{\mathrm{B}}^{-} \mathrm{m}_{\mathrm{B}}^{-}\right) \mathrm{galdcm} r \mathrm{ne} 131 \lambda(\mathrm{DE} 3)$ & Invitrogen \\
\hline E.coli DH5a & deo $R$, recA1, endA1, hsdR17(rk-,mk +), phoA, supE44, $\lambda-$, thi-1, gyrA96, relA1 & Takara \\
\hline YJM60 & E.coli BL21(DE3)/pYJM60 & This study \\
\hline YJM61 & E.coli BL21(DE3)/pYJM61 & This study \\
\hline YJM62 & E.coli BL21(DE3)/pYJM62 & This study \\
\hline YJM63 & E.coli BL21(DE3)/pYJM63, pYJM62 & This study \\
\hline YJM64 & E.coli BL21(DE3)/pYJM64, pYJM62 & This study \\
\hline YJM66 & E.coli BL21(DE3)/pYJM66, pYJM14, pYJM62 & This study \\
\hline YJM67 & E.coli BL21(DE3)/pYJM66, pYJM14, pYJM67 & This study \\
\hline \multicolumn{3}{|l|}{ Plasmids } \\
\hline pACYCDuet-1 & P15A (pACYC184), Cm & Novagen \\
\hline pTrcHis2B & pBR322 origin, Ampr & Invitrogen \\
\hline pCOLADuet-1 & ColA ori, lacl T7lac, Kan ${ }^{r}$ & Novagen \\
\hline pYJM14 & pTrcHis2B carrying ERG12, ERG8, ERG19 and IDI1 from Saccharomyces cerevisiae & {$[29]$} \\
\hline pYJM16 & pACYCDuet-1 carrying mvaE and mvas from Enterococcus faecalis & {$[28]$} \\
\hline pYJM60 & pCOLADuet-1 carrying $A C S_{E C}$ from E. coli & This study \\
\hline pYJM61 & pCOLADuet-1 carrying $A C S_{S T}$ from Salmonella typhimurium LT2 & This study \\
\hline pYJM62 & pCOLADuet-1 carrying ACS AP from Acetobacter pasteurianus & This study \\
\hline pYJM63 & pACYCDuet-1 carrying QHS1 from Artemisia annua & This study \\
\hline pYJM64 & pACYCDuet-1 carrying QHS1 from Artemisia annua, GPPS2 from Abies grandis & This study \\
\hline pYJM65 & pACYCDuet-1 carrying QHS1 from Artemisia annua, mvaE and mvas from Enterococcus faecalis & This study \\
\hline pYJM66 & $\begin{array}{l}\text { pACYCDuet-1 carrying QHS1 from Artemisia annua, mvaE and mvas from Enterococcus faecalis, } \\
\text { GPPS2 from Abies grandis }\end{array}$ & This study \\
\hline pYJM67 & 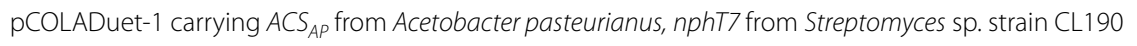 & This study \\
\hline
\end{tabular}


The geranyl diphosphate synthase (GPPS2) gene (GenBank No. AF513112) from Abies grandis was analyzed by online software (http://www.genscript.com/cgi-bin/ tools/rare_codon_analysis) and optimized to the preferred codon usage of $E$. coli (http://www.jcat.de/). The codon-optimized GPPS2 gene was synthesized by Genray Company with the plasmid pGH as the vector (named pGH-GPPS2).

To get the plasmid pYJM60 (pCOL- $A C S_{E C}$ ), pYJM61 $\left(\mathrm{pCOL}-A C S_{S T}\right), \mathrm{pYJM} 62$ (pCOL- $\left.A C S_{A P}\right)$, the $A C S_{E C}$, $A C S_{S T}$ and $A C S_{A P}$ genes were ligated into pCOLADuet-1 using NcoI and BamHI, NcoI and BamHI, BamHI and EcoRI, respectively.

The QHS1 gene fragment was obtained by digestion of pGH-QHS1 with BglII and FseI and then ligated into the corresponding sites of pACYCDuet-1 to create pYJM63 (pACY-QHS1).

To get the plasmid pYJM64, The GPPS2 gene fragment was obtained by digestion of pGH-GPPS2 with XhoI and PacI, and then ligated into the corresponding sites of pYJM63 to create pYJM64 (pACY-QHS1-GPPS2) (Fig. 5a).

To get the plasmid pYJM65, the QHS1 gene fragment was obtained by excision from pYJM63 with BglII and FseI and was introduced into the corresponding sites of pYJM16 to create pYJM65 (pACY-mvaE-mvaS-QHS1).

The GPPS2 gene fragment was obtained by digestion of pGH-GPPS2 with XhoI and PacI, and then ligated into the corresponding sites of pYJM65 to create PYJM66 (pACY-mvaE-mvaS-QHS1-GPPS2) (Fig. 5b).

To get the plasmid pYJM67, the nphT7 gene fragment was obtained by digestion of pGH-nphT7 using Nde I and Bgl II enzymes, and then ligated into the pYJM67(pCOL-

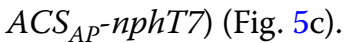

The plasmid pYJM16 was created based on pACYCDuet-1 by introducing mvaE and mvaS from Enterococcus faecalis [39]. The plasmid pYJM14 was constructed on pTrcHis2B by introducing the ERG8, ERG12, ERG19 and IDI1 from S. Cerevisiae [40].

\section{$\beta$-caryophyllene production under flask conditions}

The engineered E. coli strains was cultivated in $50 \mathrm{ml}$ fermentation medium as described above plus chloramphenicol $(34 \mu \mathrm{g} / \mathrm{ml})$ or/and ampicillin $(100 \mu \mathrm{g} / \mathrm{ml})$ or/and kanamycin $(50 \mu \mathrm{g} / \mathrm{ml})$ with shaking at $180 \mathrm{rpm}$. To induce gene expression at $30{ }^{\circ} \mathrm{C}$ for $56 \mathrm{~h}, 0.25 \mathrm{mM}$ IPTG was added into the medium when the $\mathrm{OD}_{600}$ achieved about 0.6. a $1 \mathrm{ml}$ headspace gas sample of $\beta$-caryophyllene was extracted from the culture broth based on the procedure of Reinsvold et al. [41] and measured by GC-FID. Different concentrations of $\beta$-caryophyllene produced by the strains were calculated by means of converting the GC peak area to $\mu \mathrm{g}$ or $\mathrm{mg}$ of $\beta$-caryophyllene via a calibration curve.

GC analysis was performed on an Agilent 7890A equipped with a flame ionization detector (FID) and an HP-INNOWAX column $(25 \mathrm{~m} \times 250 \mu \mathrm{m} \times 0.2 \mu \mathrm{m})$. N2 was used as carrier gas with a linear velocity of $1 \mathrm{ml} / \mathrm{min}$. The column temperature profile was $50{ }^{\circ} \mathrm{C}$ for $0.5 \mathrm{~min}$, $4{ }^{\circ} \mathrm{C} / \mathrm{min}$ increase to $70{ }^{\circ} \mathrm{C}, 15{ }^{\circ} \mathrm{C} / \mathrm{min}$ increase to $150{ }^{\circ} \mathrm{C}$, $25{ }^{\circ} \mathrm{C} / \mathrm{min}$ increase to $250{ }^{\circ} \mathrm{C}$ and $250{ }^{\circ} \mathrm{C}$ for $5 \mathrm{~min}$. The product was characterized by direct comparison with an authentic standard (Sigma-Aldrich). The peak area was converted into $\beta$-caryophyllene concentration according to a standard curve plotted with a set of known concentrations of $\beta$-caryophyllene. All the error bars presented in the results indicated the standard deviation from the mean $(\mathrm{n}=3)$.

\section{Optimization of type and concentration of organic nitrogen source Effect of type of organic nitrogen source}

The shake-flask cultures were incubated in M9 minimum medium with different organic nitrogen sources $(10 \mathrm{~g} / \mathrm{L})$ (beef extract (solarbio), beef extract (Aladdin), beef extract (Beijing Shuangxuan Microbe Culture Medium Products Factory), beef extract (Sinopharm Chemical Reagent Co., Ltd), beef extract powder (MDBio, Inc), yeast extract powder (Beijing AoBoXing Bio-Tech Co., Ltd) at the above-mentioned culture conditions, and the $\beta$-caryophyllene products were detected.

\section{Effect of concentration of organic nitrogen source}

The shake-flask cultures were incubated in M9 minimal medium with different beef extract (solarbio) concentrations $(1,5,10$ or $15 \mathrm{~g} / \mathrm{L})$ at the above- mentioned culture conditions, and the $\beta$-caryophyllene products were detected.

\section{Fed-batch fermentation}

To further enhance the $\beta$-caryophyllene production from HAc, the fed-batch fermentation was carried out in a 5-L fermentor (BIOSTAT Bplus MO5L, Sartorius, Germany). To decrease the poisonousness of HAc on cells, a $\mathrm{pH}$ coupled fed-batch fermentation was conducted as previously described by J.C. Liao [42]. The strain was grown in $2 \mathrm{~L}$ fermentation medium. The temperature was controlled at $30{ }^{\circ} \mathrm{C}$ and the $\mathrm{pH}$ was maintained at $\sim 6.0$ during the entire fermentation by adding pure HAc through an auto-pump. Cells were induced at an $\mathrm{OD}_{600}$ of $\sim 4$ using $0.25 \mathrm{mM}$ IPTG. The residual HAc was measured via an enzyme kit (R-Biopharm). Then $\beta$-caryophyllene production was determined at a series of time points by GC as stated above. The biomass was determined by 

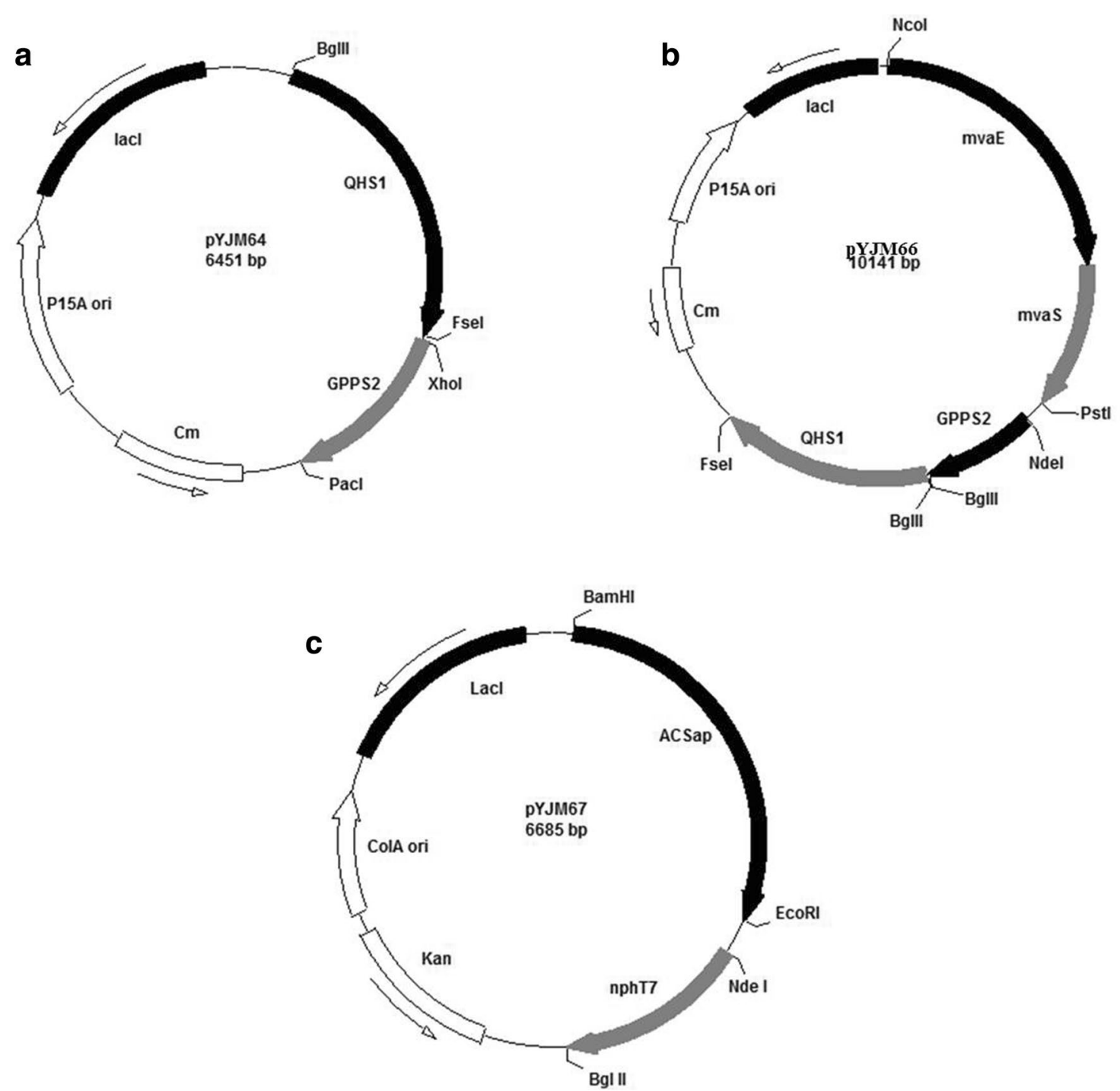

Fig. 5 Plasmids used in this study. a Represented the plasmid PYJM64 harboring QHS1 from Artemisia annua, GPPS2 from Abies grandis; b represented the plasmid pYJM66 carrying QHS1 from Artemisia annua, mvaE and mvaS from Enterococcus faecalis, GPPS2 from Abies grandis; c represented the plasmid pYJM67 containing ACS AP from Acetobacter pasteurianus, nphT7 from Streptomyces sp. strain CL190

measuring the $\mathrm{OD}_{600}$ using a spectrophotometer (Cary $50 \mathrm{UV}-$ Vis, Varian), where an $\mathrm{OD}_{600}$ of one corresponded to $0.43 \mathrm{~g}$ dry weight/L for $E$. coli BL21 strains.

Conversion efficiency (gram to gram) of HAc to $\beta$-caryophyllene was calculated with the following equation: $\mathrm{Y}=\mathrm{G}_{\mathrm{c}} / \mathrm{G}_{\mathrm{h}} \times 100 \%$.

Where $\mathrm{Y}=$ conversion efficiency (gram to gram, $100 \%) ; G_{c}=$ weight of $\beta$-caryophyllene $(\mathrm{g}) ; \mathrm{G}_{\mathrm{h}}=$ weight of HAc (g).

\section{Abbreviations}

DMAPP: dimethylallyl pyrophosphate; IPP: isopentenyl pyrophosphate; GPP: geranyl diphosphate; MVA: mevalonate; IPTG: isopropyl $\beta$-D-thiogalactoside; PCR: polymerase chain reaction; GC: gas chromatography; FPP: farnesyl diphosphate.

\section{Authors' contributions}

JY developed the idea for the study. JY designed the research, did the literature review and prepared the manuscript. QN helped to revise the manuscript. All authors read and approved the final manuscript.

\section{Author details}

${ }^{1}$ Key Lab of Plant Biotechnology in Universities of Shandong Province; College of Life Sciences, Qingdao Agricultural University, Qingdao 266109, China.

${ }^{2}$ Key Lab of Applied Mycology, College of Life Sciences, Qingdao Agricultural University, No.700 Changcheng Road, Chengyang District, Qingdao 266109, China. ${ }^{3}$ Foreign Languages School, Qingdao Agricultural University, Qingdao 266109, China.

\section{Acknowledgements}

This work was financially supported by the National Natural Science Foundation of China (Grant No. 21572242), the Natural Science Foundation of Shandong Province, China (Grant No. ZR2015BM021), the Recruitment Program of High-end Foreign Experts of the State Administration of Foreign Experts Affairs (Grant NO. GDW20153500203), the project of Science and Technology for 
People's livelihood of Qingdao (No. 15-9-2-94-nsh), the special project of science and technology development for construction (Grant No. JK2015-22), the Talents of High Level Scientific Research Foundation (Grant No. 6631113326) of Qingdao Agricultural University, the National Natural Science Foundation of China (Grant No. 31300599), the Talents of High Level Scientific Research Foundation (Grant No. 6631113318) of Qingdao Agricultural University and the National Natural Science Foundation of China (Grant No. 31172012/c1506).

\section{Competing interests}

The authors declare that they have no competing interests.

Received: 28 January 2016 Accepted: 27 April 2016

Published online: 05 May 2016

\section{References}

1. Knudsen JT, Tollsten L, Bergström LG. Floral scents-a checklist of volatile compounds isolated by head-space techniques. Phytochemistry. 1993:33(2):253-80.

2. Kubo I, Chaudhuri SK, Kubo Y, Sanchez Y, Ogura T, Saito T, Ishikawa H, Haraguchi H. Cytotoxic and antioxidative sesquiterpenoids from Heterotheca inuloides. Planta Med. 1996;62(5):427-30.

3. Meylemans HA, Quintana RL, Harvey BG. Efficient conversion of pure and mixed terpene feedstocks to high density fuels. Fuel. 2012;97:560-8.

4. Harvey BG, Meylemans HA, Gough RV, Quintana RL, Garrison MD, Bruno TJ. High-density biosynthetic fuels: the intersection of heterogeneous catalysis and metabolic engineering. Phys Chem Chem Phys. 2014;16(20):9448-57.

5. Ryder JA. Limonane (p-menthane) and farnesane; jet biofuel. In.: Google Patents; 2009.

6. Maury J, Asadollahi MA, Møller K, Clark A, Nielsen J. Microbial isoprenoid production: an example of green chemistry through metabolic engineering. Adv Biochem Eng Biotechnol. 2005;100:19-51.

7. Chang MC, Keasling JD. Production of isoprenoid pharmaceuticals by engineered microbes. Nat Chem Biol. 2006;2(12):674-81.

8. Mazumdar S, Clomburg JM, Gonzalez R. Escherichia coli strains engineered for homofermentative production of D-lactic acid from glycerol. Appl Environ Microbiol. 2010;76(13):4327-36.

9. Wolfe AJ. The acetate switch. Microbiol Mol Biol Rev. 2005;69(1):12-50.

10. Fleisch T, Sills R, Briscoe M. A review of global GTL developments. J Nat Gas Chem. 2002;11:1-14.

11. Henstra AM, Sipma J, Rinzema A, Stams AJ. Microbiology of synthesis gas fermentation for biofuel production. Curr Opin Biotechnol. 2007:18(3):200-6.

12. Munasinghe PC, Khanal SK. Biomass-derived syngas fermentation into biofuels: opportunities and challenges. Bioresour Technol. 2010;101(13):5013-22.

13. Ruan Z, Zanotti M, Wang X, Ducey C, Liu Y. Evaluation of lipid accumulation from lignocellulosic sugars by Mortierella isabellina for biodiesel production. Bioresour Technol. 2012;110:198-205.

14. Zhang Y-HP: Reviving the carbohydrate economy via multi-product lignocellulose biorefineries. J Ind Microbiol Biotechnol. 2008; 35(5):367-75.

15. Periana RA, Mironov O, Taube D, Bhalla G, Jones C. Catalytic, oxidative condensation of $\mathrm{CH} 4$ to $\mathrm{CH} 3 \mathrm{COOH}$ in one step via $\mathrm{CH}$ activation. Science. 2003;301(5634):814-8.

16. Chen Y, Cheng JJ, Creamer KS. Inhibition of anaerobic digestion process: a review. Bioresour Technol. 2008;99(10):4044-64.

17. Chi Z, Zheng Y, Ma J, Chen S. Oleaginous yeast Cryptococcus curvatus culture with dark fermentation hydrogen production effluent as feedstock for microbial lipid production. Int J Hydrogen Energy. 2011;36(16):9542-50

18. Lian J, Garcia-Perez M, Coates R, Wu H, Chen S. Yeast fermentation of carboxylic acids obtained from pyrolytic aqueous phases for lipid production. Bioresour Technol. 2012;118:177-86.

19. Christophe G, Deo JL, Kumar V, Nouaille R, Fontanille P, Larroche C. Production of oils from acetic acid by the oleaginous yeast Cryptococcus curvatus. Appl Biochem Biotechnol. 2012;167(5):1270-9.

20. Chen C-K, Blaschek HP. Effect of acetate on molecular and physiological aspects of Clostridium beijerinckii NCIMB 8052 solvent production and strain degeneration. Appl Environ Microbiol. 1999:65(2):499-505.
21. Canganella F, Kuk S-U, Morgan H, Wiegel J. Clostridium thermobutyricum: growth studies and stimulation of butyrate formation by acetate supplementation. Microbiol Res. 2002;157(2):149-56.

22. Krivoruchko A, Zhang Y, Siewers V, Chen Y, Nielsen J. Microbial acetyl-CoA metabolism and metabolic engineering. Metab Eng. 2015;28:28-42.

23. Lin H, Castro NM, Bennett GN, San K-Y. Acetyl-CoA synthetase overexpression in Escherichia coli demonstrates more efficient acetate assimilation and lower acetate accumulation: a potential tool in metabolic engineering. Appl Microbiol Biotechnol. 2006;71(6):870-4.

24. Zha W, Rubin-Pitel SB, Shao Z, Zhao H. Improving cellular malonylCoA level in Escherichia coli via metabolic engineering. Metab Eng. 2009;11(3):192-8.

25. Yan Y, Liao JC. Engineering metabolic systems for production of advanced fuels. J Ind Microbiol Biotechnol. 2009;36(4):471-9.

26. Sun $X$, Shen $X$, Jain R, Lin Y, Wang J, Sun J, Wang J, Yan Y, Yuan Q. Synthesis of chemicals by metabolic engineering of microbes. Chem Soc Rev. 2015:44(11):3760-85

27. Lin Y, Jain R, Yan Y. Microbial production of antioxidant food ingredients via metabolic engineering. Curr Opin Biotechnol. 2014;26:71-8.

28. Cai Y, Jia J-W, Crock J, Lin Z-X, Chen X-Y, Croteau R. A cDNA clone for $\beta$-caryophyllene synthase from Artemisia annua. Phytochemistry. 2002:61 (5):523-9.

29. Jayaramaiah $\mathrm{RH}$, Anand A, Beedkar SD, Dholakia BB, Punekar SA, Kalunke RM, Gade WN, Thulasiram HV, Giri AP. Functional characterization and transient expression manipulation of a new sesquiterpene synthase involved in $\beta$-caryophyllene accumulation in Ocimum. Biochem Biophys Res Commun. 2016:473:265-71.

30. Burke C, Croteau R. Geranyl diphosphate synthase from Abies grandis: cDNA isolation, functional expression, and characterization. Arch Biochem Biophys. 2002;405(1):130-6

31. Yang J, Nie Q, Ren M, Feng H, Jiang X, Zheng Y, Liu M, Zhang H, Xian M. Metabolic engineering of Escherichia coli for the biosynthesis of alphapinene. Biotechnol Biofuels. 2013;6(1):60.

32. Zhang H, Liu Q, Cao Y, Feng X, Zheng Y, Zou H, Liu H, Yang J, Xian M. Microbial production of sabinene-a new terpene-based precursor of advanced biofuel. Microb Cell Fact. 2014;13(20):3826-31.

33. Morrone D, Lowry L, Determan MK, Hershey DM, Xu M, Peters RJ. Increasing diterpene yield with a modular metabolic engineering system in E. coli: comparison of MEV and MEP isoprenoid precursor pathway engineering. Appl Microbiol Biotechnol. 2010;85(6):1893-906.

34. Vadali RV, Fu Y, Bennett GN, San KY. Enhanced lycopene productivity by manipulation of carbon flow to isopentenyl diphosphate in Escherichia coli. Biotechnol Prog. 2005;21(5):1558-61.

35. MATSUMOTO Ki, Yamada M, Leong CR, Jo S-J, Kuzuyama T, Taguchi S: A new pathway for poly (3-hydroxybutyrate) production in Escherichia coli and Corynebacterium glutamicum by functional expression of a new acetoacetyl-coenzyme A synthase. Biosci Biotechnol Biochem 2011; 75(2):364-6.

36. Okamura E, Tomita T, Sawa R, Nishiyama M, Kuzuyama T. Unprecedented acetoacetyl-coenzyme A synthesizing enzyme of the thiolase superfamily involved in the mevalonate pathway. Proc Natl Acad Sci. 2010;107(25):11265-70.

37. Torija MaJ, Beltran G, Novo M, Poblet M, Rozès N, Guillamón JM, Mas A: Effect of the nitrogen source on the fatty acid composition of Saccharomyces cerevisiae. Food microbio/ 2003; 20(2):255-8.

38. Yang J, Guo L. Biosynthesis of $\beta$-carotene in engineered E. coli using the MEP and MVA pathways. Microb Cell Fact. 2014;13(1):1-11.

39. Yang J, Xian M, Su S, Zhao G, Nie Q, Jiang X, Zheng Y, Liu W. Enhancing production of bio-isoprene using hybrid MVA pathway and isoprene synthase in E. coli. PLoS One. 2012;7(4):e33509.

40. Yang J, Zhao G, Sun Y, Zheng Y, Jiang X, Liu W, Xian M. Bio-isoprene production using exogenous MVA pathway and isoprene synthase in Escherichia coli. Bioresour Technol. 2012;104:642-7.

41. Reinsvold RE, Jinkerson RE, Radakovits R, Posewitz MC, Basu C. The production of the sesquiterpene $\beta$-caryophyllene in a transgenic strain of the Cyanobacterium synechocystis. J Plant Physiol. 2011;168(8):848-52.

42. Li H, Opgenorth PH, Wernick DG, Rogers S, Wu T-Y, Higashide W, Malati P, Huo Y-X, Cho KM, Liao JC. Integrated electromicrobial conversion of $\mathrm{CO}_{2}$ to higher alcohols. Science. 2012;335(6076):1596. 